\title{
On the Zeros of the Incomplete Gamma Function
}

\author{
By K. S. Kölbig
}

\begin{abstract}
Some asymptotic formulae given elsewhere for the zeros of the incomplete gamma function $\gamma(a, x)$ are corrected. A plot of a few of the zero trajectories of the function $\gamma(x w, x)$ is given, where $x$ is a real parameter. Based on theoretical work by Mahler, it is seen that the zero trajectories of $\gamma(x w, x)$ lie in a finite region of the complex $w$-plane.
\end{abstract}

1. Introduction. Let $a=\alpha+i \beta$ be a complex variable. The incomplete gamma function $\gamma(a, x)$ can then be defined by [1]

$$
\begin{aligned}
\gamma(a, x) & =\int_{0}^{x} t^{a-1} e^{-t} d t & & (\alpha>0) \\
& =\sum_{n=0}^{\infty} \frac{(-1)^{n}}{n !} \frac{x^{a+n}}{a+n} & & (a \neq 0,-1,-2, \cdots) .
\end{aligned}
$$

The zeros of this function have been treated theoretically by several authors, for example, by Franklin [2], Walther [3], Rasch [4], Hille and Rasch [5], Mahler [6], and Tricomi [7], [8], [9]; some of the papers seem to be little known. Numerical values for complex zeros have been given by Franklin [2] for $\gamma(a, 1)$, and more recently by Kölbig [10], [11], who calculated the first eight zero trajectories of $\gamma(a, x)$ in the $a$-plane as functions of $x>0$.

It is the aim of this note to recall some of the earlier results (occasionally correcting them), and to present a plot containing a few of the zero trajectories of the function $\gamma(x w, x)$ in the complex $w$-plane $(w=u+i v)$, as functions of the real parameter $x>0$. It will be seen that these trajectories all lie in a finite region of the $w$-plane, and that they cluster towards a limiting curve as shown by Mahler [6], who investigated the function $\gamma(x w, x)$ in detail.

2. Some Results on the Zeros of $\gamma(\alpha, x)$. From the results obtained by the authors mentioned above, we recall here that

(a) for real $x \neq 0$ and real $a=\alpha$, the function $\gamma(\alpha, x)$ has no zero if $\alpha>0$, a negative zero $x_{0}^{-}(\alpha)$ if $\alpha<0, \alpha \neq-1,-2,-3, \cdots$, and, in addition to $x_{0}^{-}(\alpha)$, a positive zero $x_{0}^{+}(\alpha)$ if $-2 m<\alpha<1-2 m(m=1,2,3, \cdots)$ [7], [8], [9]. The investigations of Tricomi [7], [8] show that the asymptotic expressions

$$
\bar{x}_{0}^{-}(\alpha)=-(1-\alpha)\left[1+\left(\frac{2}{1-\alpha}\right)^{1 / 2} y_{0}(\alpha)\right]+O\left(|\alpha|^{-1 / 2}\right)
$$

and

$$
x_{0}^{+}(\alpha)=-\tau \alpha-\frac{\tau}{1+\tau} \log \frac{(1+\tau)[(1-\alpha) \pi / 2]^{1 / 2}}{\sin \alpha \pi}+O\left(\alpha^{-2} \log ^{2}|\alpha|\right)
$$

Received October 26, 1971.

AMS 1969 subject classifications. Primary 3315, 6525.

Key words and phrases. Incomplete gamma function, complex zeros.

Copyright $@$ ( 1972, American Mathematical Society 
hold for $\alpha \rightarrow-\infty$, provided the value of $\alpha$ is not too close to a negative integer. $y_{0}(\alpha)$ is the real root of the equation

$$
\operatorname{Erfi}(y)=\frac{1}{2} \sqrt{ } \pi \cot |\alpha| \pi,
$$

where

$$
\operatorname{Erfi}(y)=-i \operatorname{Erf}(i y)=\int_{0}^{y} e^{t^{2}} d t
$$

is the error function with imaginary argument. $\tau=0.278464542761074$ is the real root of the equation

$$
1+t+\log t=0 .
$$

It should be noted that the above formulae (2), (3), (4) differ from the three disparate versions of these formulae given originally in [7], [8], [9]. The corrected versions given above may be obtained by reworking the original proofs. In particular, in the relevant formulae (1) and (2) on p. 187, and (7) on p. 190 of [8], the variable $\alpha$ should be replaced by $1-\alpha$, and not by $|\alpha|$ as has been done in [9], where this procedure has caused an additional sign error to be introduced in formula (3) on p. 63.*

(b) For complex $a=\alpha+i \beta$ and fixed real $x \leqq x_{1}^{*}=0.308089926457654$, $\gamma(a, x)$ has only real zeros, which lie on the negative $\alpha$-axis [5], [6].

(c) For complex $a$ and fixed real $x>x_{1}^{*}, \gamma(a, x)$ has, in addition to the real zeros on the negative $\alpha$-axis, at least one pair of complex conjugate zeros $a^{0}(x)$, and at most a finite number $M(x)$ pairs of such zeros [5], [6].

As functions of $x$, the zeros of $\gamma(a, x)$ represent trajectories in the $a$-plane. Since these zeros are conjugate complex, one can restrict the problem to the upper halfplane $\beta \geqq 0$. As explained in [10], these trajectories start at points $\alpha_{m}^{*}(m=1,2,3, \cdots)$ on the negative $\alpha$-axis for certain values $x_{m}^{*} \geqq x_{1}^{*}$. For increasing $x>x_{m}^{*}$ they continue smoothly through the upper half-plane $\beta>0$ and tend to infinity as shown for some of them in [10], [11]. For $m \rightarrow \infty$, the following asymptotic expressions can be found, for $\alpha_{m}^{*}$ and $x_{m}^{*}$,

$$
\begin{aligned}
\alpha_{m}^{*} & =1-2 m-\frac{1}{2}-\frac{1}{\pi} \arctan \frac{1+\tau}{\pi}+O\left(m^{-1}\right) \\
& \sim 1-2 m-0.623021
\end{aligned}
$$

or $\alpha_{m}^{*} \sim-2 m+\delta$ with $\delta=0.376979$, and

$$
\begin{aligned}
x_{m}^{*} & =2 \tau m-\frac{1}{2} \frac{\tau}{1+\tau} \log m-\tau \delta+\frac{\tau}{1+\tau} \log \left(\frac{1}{1+\tau} \frac{\sin \delta \pi}{\sqrt{ } \pi}\right)+O\left(\frac{\log ^{2} m}{m}\right) \\
& \sim 0.556929 m-0.108906 \log m-0.299840 .^{* *}
\end{aligned}
$$

* Note that the incorrect formulae corresponding to (2), (3) and (4) have been propagated from [7] into the Bateman project handbook of higher transcendental functions [12, p. 141], with an additional misprint in (4).

** The constant given by the author in the corresponding formula (25) of [10] is incorrect, due to the use of one of the erroneous versions of (3). For the same reason, Eq. (23) of [10] should be replaced by $\frac{1}{2}(1-s)^{-1}+\pi \cot \pi s=1+\tau$. 
By comparison with the numerical values in Table 1 below, one sees that for $m=5$ the relative error of (7) is less than $0.1 \%$, whereas the relative error of $(8)$ is less than $1.2 \%$. The above formulae can be deduced by differentiation from (3). These formulae are also given by Rasch [4] (with a weaker $O$-term iñ (7)) and, in part, by Mahler [6]. Rasch [4] also gives an asymptotic expression, obtainable from (8), for the number of pairs of complex conjugate zeros for $x \rightarrow \infty$, namely

$$
\begin{aligned}
& M(x)= {\left[\frac{1}{2 \tau} x+\frac{1}{4} \frac{1}{1+\tau} \log x\right.} \\
&\left.+\frac{1}{2} \delta-\frac{1}{2} \frac{1}{1+\tau} \log \left(\frac{(2 \tau)^{1 / 2}}{1+\tau} \frac{\sin \delta \pi}{\sqrt{ } \pi}\right)+O\left(\frac{\log ^{2} x}{x}\right)\right]+\epsilon \\
& \sim[1.795561 x+0.195547 \log x+0.652837]+\epsilon,
\end{aligned}
$$

where $\epsilon$ can take the values $-1,0,1$, and where $[\xi]$ is the integer part of $\xi$.

3. A Plot of the Zeros of $\gamma(x w, x)$. From the results of Mahler [6] and Kölbig [10], one can see that for real $x=x_{m}^{*}$ the zero trajectories $w_{m}^{0}(x)$ in the upper half-plane $v \geqq 0$ start on the negative $u$-axis at the points $u_{m}^{*}=\alpha_{m}^{*} / x_{m}^{*}$, where $u_{m+1}^{*}>u_{m}^{*}(m=1$, $2,3, \cdots)$. For increasing $x$, they continue through the half-plane $v>0$ as roughly semilenticular trajectories. As $x \rightarrow \infty$ they approach the point $w=1$ for all $m$. As shown by Mahler [6], for $m \rightarrow \infty$ these curves cluster towards the limiting curve $w_{\infty}^{0}$ defined by

$$
\operatorname{Re}\{w \log w-w+1\}=0 \quad(u \leqq 1)
$$

or

$$
\frac{1}{2} u \log \left(u^{2}+v^{2}\right)-v \arctan (v / u)-u+1=0 \quad(u \leqq 1) .
$$

This curve starts on the negative $u$-axis at the point $u_{\infty}^{*}=-1 / \tau=-3.591121$, in agreement with the limiting value for $u_{\infty}^{*}$ obtained from (7) and (8). It increases up to its maximum value at the coordinates $(U, V)$, crosses the $v$-axis at $v=2 / \pi$, and reaches the positive $u$-axis at $u=1$. By differentiation one finds that

$$
v^{\prime}(u, v)=\frac{d v}{d u}=\frac{1}{2} \frac{\log \left(u^{2}+v^{2}\right)}{\arctan (v / u)} .
$$

In particular, for the slope at the endpoints, we have

$$
v^{\prime}\left(u_{\infty}^{*}, 0\right)=(1+\tau) / \pi=0.406948 \text { and } v^{\prime}(1,0)=-1 .
$$

For the coordinates of the maximum value, we obtain $U=-0.689158$ and $V=$ 0.724611 , where $U$ is the negative root with smallest modulus of the equation

$$
U=\cos \left(\frac{1-U}{1+U}\right)^{1 / 2}
$$

and $V=\left(1-U^{2}\right)^{1 / 2}$.

Table 1, which is calculated in part from Table 2 of [10], shows the starting points $u_{m}^{*}$ of the first ten zero trajectories of $\gamma(x w, x)$, together with the values of $x_{m}^{*}$ and $\alpha_{m}^{*}$. 


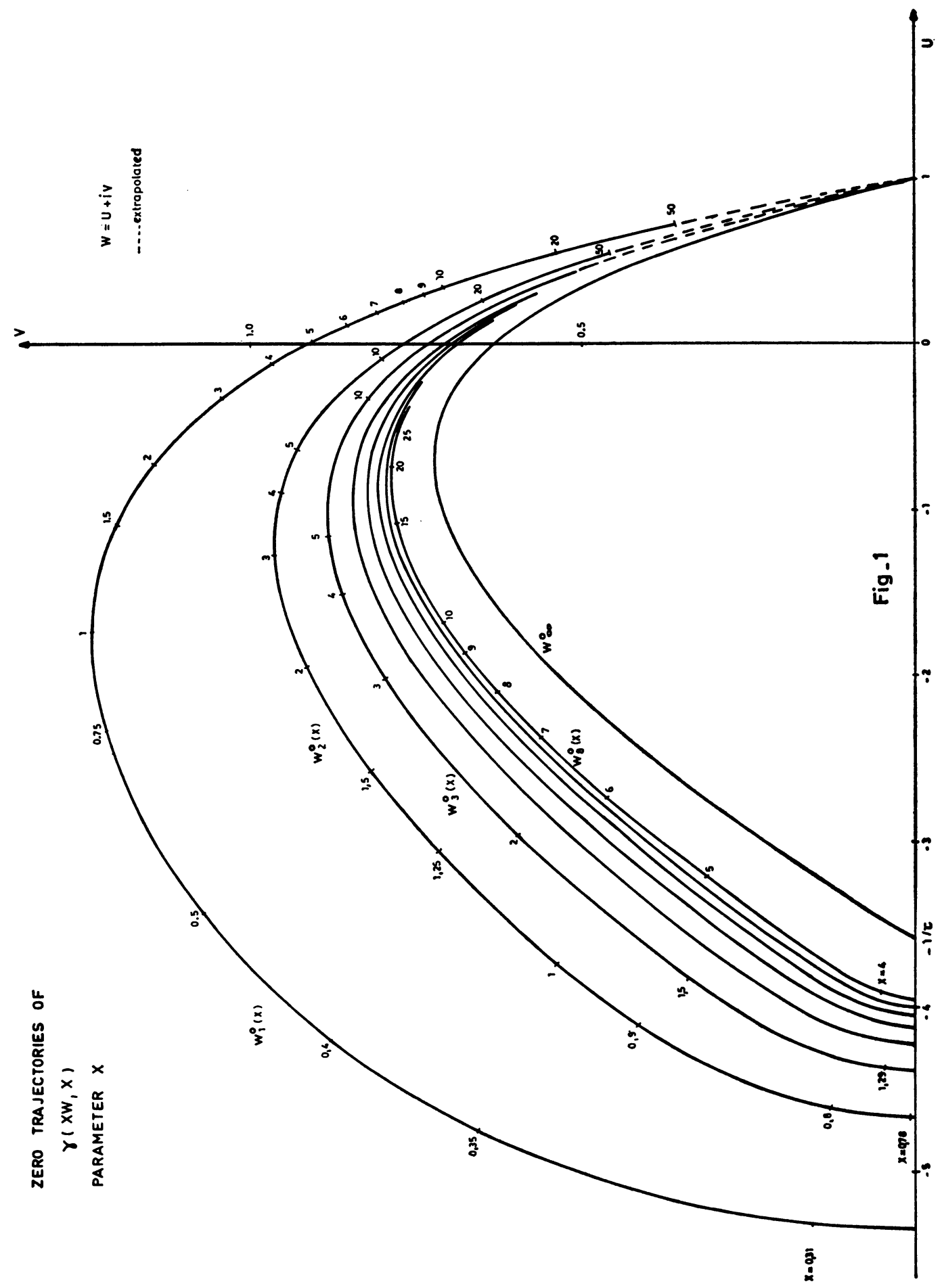


TABLE 1

\begin{tabular}{cccc}
\hline$m$ & $x_{m}^{*}$ & $\alpha_{m}^{*}$ & $u_{m}^{*}$ \\
\hline 1 & 0.30809 & -1.64425 & -5.33690 \\
2 & 0.77997 & -3.63887 & -4.66539 \\
3 & 1.28634 & -5.63573 & -4.38120 \\
4 & 1.80754 & -7.63372 & -4.22327 \\
5 & 2.33692 & -9.63230 & -4.12179 \\
6 & 2.87150 & -11.63126 & -4.05059 \\
7 & 3.40964 & -13.63044 & -3.99762 \\
8 & 3.95039 & -15.62979 & -3.95652 \\
9 & 4.49311 & -17.62925 & -3.92362 \\
10 & 5.03739 & -19.62880 & -3.89662 \\
\hline
\end{tabular}

Figure 1 shows the plot of the first eight trajectories. These curves have been calculated on a CDC 6600 computer in essentially the same way as described in [10]. Because of the increasing computational difficulties for $x \rightarrow \infty$, the curves have been extrapolated near $w=1$. All the trajectories lie between $w_{1}^{0}(x)$ and $w_{\infty}^{0}$ and are symmetric with respect to the $u$-axis.

We add here that Mahler [6] has also treated the behaviour of the zeros of $\gamma(x w, x)$ in the case of complex $x=|x| e^{i \phi}$, where $|\phi|<\pi / 2$. He has also investigated the problem of the zeros of the complementary incomplete gamma function $\Gamma(x w, x)=$ $\Gamma(x w)-\gamma(x w, x)$.

\section{CERN}

1211 Geneva 23

Switzerland

1. M. Abramowitz \& I. A. Stegun (Editors), Handbook of Mathematical Functions, With Formulas, Graphs, and Mathematical Tables, 5th printing with corrections, Nat. Bur. Standards Appl. Math. Series, 55, U.S. Government Printing Office, Washington, D. C., 1966. MR 34 \#8607.

2. P. Franklin, "Calculation of the complex zeros of the function $P(z)$ complementary to the incomplete gamma function," Ann. of Math. (2), v. 21, 1919, pp. 61-63.

3. A. WALTHER, "Über die reellen Nullstellen der unvollständigen Gammafunktion $P(z)$," Math. Z., v. 23, 1925, pp. 238-245.

4. G. RASCH, "Öber die Nullstellen der unvollständigen Gammafunktion $P(z, \rho)$. I. Die reellen Nullstellen von $P(z, \rho)$ bei positivem reellen $\rho$," Math. Z., v. 29, 1929, pp. 301-318.

5. E. HiLle \& G. RASCH, "Über die Nullstellen der unvollständigen Gammafunktion $P(z, \rho)$. II. Geometrisches über die Nullstellen," Math. Z., v. 29, 1929, pp. 319-334.

6. K. MaHLER, "Ueber die Nullstellen der unvollstaendigen Gammafunktion," Rend. Circ. Mat. Palermo, v. 46, 1930, pp. 1-42.

7. F. G. Tricomi, "Asymptotische Eigenschaften der unvollständigen Gammafunktion," Math. Z., v. 53, 1950, pp. 136-148. MR 13, 553.

8. F. G. TrICOMI, Funzioni ipergeometriche confluenti, Edizioni Cremonese, Roma, 1954. MR 17, 967.

9. F. G. Tricomi, Fonctions hypergéométriques confluentes, Mémor. Sci. Math., fasc. 140, Gauthier-Villars, Paris, 1960. MR 22 \#11163.

10. K. S. KölBIG, "Complex zeros of an incomplete Riemann zeta function and of the incomplete gamma function," Math. Comp., v. 24, 1970, pp. 679-696. MR 42 \#8663.

11. K. S. KöLbIG, "Complex zeros of two incomplete Riemann zeta functions," Math. Comp., v. 26, 1972, pp. 551-565.

12. A. ERdélyi, W. Magnus, F. Oberhettinger \& F. G. Tricomi, Higher Transcendental Functions. Vol. II, McGraw-Hill, New York, 1953. MR 15, 419. 